\title{
Morality with an Accent
}

ABSTRACT: In this paper, the difficulties inherent in the debate between moral nativists and antinativists, who differ in their beliefs on the nature of systems of morality, are shown to exemplify the need for philosophers to support their views with empirical data. Furthermore, it proposes that the need for phirical study of first-generation immigrant populations has the potential to resolve the debate over moral nativism, as it would allow researchers to observe the moral "critical period." Based on the recent philosophical advances made through experimental evidence, this paper goes on to argue that empirical data is a valuable source of information from which philosophers ought to draw.

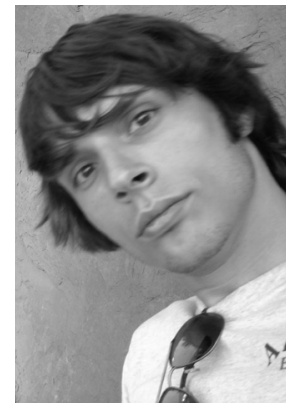

Douglas Romney is a senior Philosophy major, Chemistry minor at the University of North Carolina at Chapel Hill. He will attend the University of North Carolina at Chapel Hill School of Medicine beginning in Fall 2009. His primary philosophical interests are in ethics, experimental philosophy, and bioethics. His primary medical interests are in psychiatry and neurology. He recently presented a paper on racial healthcare disparities at the 2009 ASBH National Undergraduate Bioethics Conference hosted by Harvard University. Douglas was awarded a university sponsored Undergraduate Research Fellowship for Summer 2008, which allowed him to pursue independent research in experimental philosophy.

magine a woman's husband was injured

in a motorcycle accident and became

paralyzed from the waist down. The wife merriage were disappointed. For the rest of his life, her husband would require a lot of $c$ care and would be depressed and inactive. But even though the wife felt very unfulfilled by her marriage, she decided not to leave him because she felt that if she did, his life would be even worse

this story, neglecting her own physical and motional needs out of a sense of obligation These feelings just come naturally, provided the reader is American. To an Indian reader howerer, it is obvious that the wife is a contented woman, exhibiting admirable self control, and quite satisfied by fulfilling her duty to her husband under trying circumstances. ${ }^{1}$ Unlike philosophers, psychologists and

sociologists have been documenting variability

The reader's heart goes out to the wife in Philosophers have recently begun to review 
the results of these studies in hopes of gaining capability, the combination of which is an valuable insight into the nature of morality. argument for nativism. The first is that if As they have done so, many have found language is learned like other skills, using the information valuable and have gone on general learning capabilities, then children to perform experiments of their own. When would require examples of incorrect grammar combined with ration arguments, psychological known as negative evidence, in addition combinedwith rationalargum experimentation provides philosophers a to correct speech to acquire command of a powerful new tool with the ability to resolve language. Chomsky's second observation is many long standing debates in ethics.

One issue to which philosophers have applied experimental methods regards the development of morality. It has long been assumed that children gain the ability to think and behave ethically through experience and some philosophers have argued that morality is "native" or innate to mankind, programmed is "native" or innate to
into the human brain.

into the human brain.
Experimental philosophy is particularly well suited to provide a solution to the debate over moral nativism. This paper will briefly summarize the recent history of the debate over nativism, with a focus on the transfer of the theory from linguistics to ethics. Relevan experimental studies and results contributing to the debate in ethics will be summarized. Finally, a new experiment with the potential to end the nativist/antinativist debate will be proposed.

\section{Developments in Linguistics}

Contemporary moral nativism grows out of a movement in linguistics which began with the work done by Dr. Noam Chomsky in the 1950's. Chomsky made several observations about child development and linguistic that children only receive examples of correc language usage in their daily encounters. The third is that children learn languages. Thus children must not learn language using generalized learning capabilities. Rather Chomsky proposed that there is a "Universal Grammar" or set of grammatical principles, univers to all languges and inciples, mankind, without which language acquisition ould be impossible.

Further research by proponents of nativis theory has led to the conclusion that there is a critical period for language acquisition. During that time, generally believed to be the first few years of life, a person develops language skills with relative ease when exposed to appropriate stimuli. If however, the child has no exposure to language dit to language during this period, they will never be able to develop a command of language, despite later exposure. ${ }^{3}$

Antinativist theories reject Chomsky's account, regarding language acquisition to be the result of more general cognitive processes, rather

than that of an otherwise inaccessible system evoted exclusively to language acquisition. There are a variety of theo catheries which fall into this cholars have made great effort to discredit Chomsky and post-Chomsky

2. Chomsky, Noam (1965). Aspects of the Theory of Syntax. MIT Press. 2. Chomsky, Noam (1965). Aspects of the Theory of Syntax. Mit Press.
P.enneberg, Eric, (1964). "The Capacity of Language Acquisition." In Fodor, Jerry and Jerrold Katz, (Eds.), The Structure of Language.
Prentice Hall. theories of language acquisition either by a Chomsky's concept of innate morality solves priori reasoning or by drawing on experimental many problems. For example, the existence evidence to negate Chomsky's claims. Much of variation is difficult to explain if morality of the work has focused on discrediting the is determined biologically. Part of the great premise that children do not receive negative appeal of using the "linguistic analogy," as it evidence. Pullum, for example, argues that came to be known, is the ability of a "Universal negative evidence is plentiful and learning is Mora possible without the need for a system devoted to language acquisition. ${ }^{4}$

\section{Application to Morality}

The rapid progress in linguistics due to the work of Chomsky did not go unnoticed by those in other disciplines. In the field of ethics, Chomsky's theory was seized upon and adapted in support of moral nativism. Three scholars working independently applied Chomsky's working independently applied Chomsky's morality. Gilbert Harman, ${ }^{5}$ Susan Dwyer, ${ }^{6}$ and John Mikhail ${ }^{7}$ all proposed that perhaps morality, like language, is acquired through some innate universal system. Like Chomsky, these academics proposed that, rather than guiding us towards a specific moral code, the "Universal Moral Grammar" provides the mental framework upon which a variety of ethical theories may be built.

For those who believe that there is a biological For those who believe that there is a biological
cause for the development of moral thought, a universal biological basis. ${ }^{6}$

Since moral nativism posits a complex biological system with the purpose of enabling moral thought, attempts must be made both to provide a mechanism by which such a system would have evolved, as well as to prove the current existence of such a system. Tracing the day is imposs pre, so a day is impossible, so attempts to provide an often presented on theoretical grounds. Such defenses attempt to provide reason to believe that the ability to moralize would be favorable to reproduction and thus evolutionarily selected. Many defenses of this type use game theory to in everyday life, the ability to moralize provides some stable, long-term strategic advantage. ${ }^{8}$

Antinativists are understandably suspicious
Moral Grammar" to explain the great variation
in morals between cultures while still asserting generations of proto-huma to the prough show that in situations that parallel those faced of such schemes. They argue that the mere

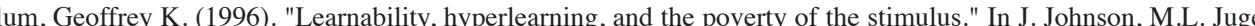
4. Pullum, Geoffrey K. (1996) "Learnability, hyperlearning, and the poverty of the stimulus." In J. Johnson, M.L. Juge
and J.L. Moxley (Eds.) Proceedings of the 22nd Annual Meeting of the Berkley Linguistics Society: General Session and Parasession on the Role of Learnability in Grammatical Theory, 498-513. Berkeley, California. 5. Harman, G. (1999). "Moral philosophy and linguistics." In K. Brinkmann (Ed.), Proceedings of the 20th World Congress of Philosophy: Volume 1: Ethics. Philosophy Documentation Center, 107-115. Reprinted in Explaining Value,

6. Dwyer, S. (1999). Mora competence. In K. Murasugi and R. Stainton (Eds.). Philosophy and Linguistics. Westview Press.
7. Mikhail, J., Sorentino, C., and Spelke, E. (1998). "Toward a universal moral grammar." In M. Gernsbacher and S. Derry, (Eds.), Proceedings, Twentieth Annual Conference of the Cognitive Science Society, Lawrence Erlbaum Associates. 8. Sripada, Chandra Sekhar (2005). Punishment and the strategic structure of moral systems Biology and Philosophy
$20: 767-789$ 
possibility of advantage in the context of a simple game does not imply the reality of the biologica evolution of morality. Rather, they have attempted to provide explanations of how mankind could have evolved to its present condition without the need for a complex biological moral system.

Along with Chomsky's theory, nativists also adopted some of his methodology, relying on empirical data to confound antinativists. Man empirical studies performed by nativists show results which are difficult to explain unde existing alternate theories. One such stud used a test known as the Wason selection task. Subjects are told that if a card shows an even number on one face, its opposite face should have a primary color. They are then asked to choose which of four cards (showing 3, 8, red, and brown) must be turned over to verify that the rule is being followed. Few subjects are able to successfully solve this logic puzzle; however, when the same problem is presented in a social context, such as checking identification at a ba and the cards replaced with people and drinks, nearly all subjects are able to solve the puzzle. This result seems to indicate that there is some This result seems to in biological system whose task it is to deal with situations of social interaction. ${ }^{10}$

Some nativists have extended the linguistic analogy so far as to posit a critical period for the acquisition of morality. During this stage, the mind would be specially equipped to absorb ethical principles. Finding such a period hardwired into our biology would provide strong evidence in favor of moral nativism. Of course, the existence of such a period is hotly

contested; however, at least one major world culture firmly believes in its existence.

The Chinese government embraces mora nativism. The current Chinese educationa system, in fact, depends on its existence. They define the moral critical period to be "a period dine the friod moral character or a period of time, in which moral character may proceed in the mos positive direction and gain best achievements, resulting from good educational conditions." For Chinese children, this age has been determined to be between seven and nine years old. During this period, Chinese educators focus on instilling "positive" moral values into their pupils so that they will exhibit desired behaviors automatically as they mature

While the Chinese themselves draw short of claiming that the effects of moral indoctrination during the critical period are permanent, or at least nearly so, this is clearly the purpose of devoting valuable educational resources to mora education during this period. Indeed, it is the effectiveness of training during this period which distinguishes it as a developmental stage. Beyond this, the linguistic anagy would indicc indicate that training during the critical period for morality

\section{Moving Forward}

It should be clear from the preceding discussion that empirical data can and does have an important role to play in philosophy. Although the general focus of ethics is to provide an account of how things ought to be much is to be gained by understanding how things actually are. Particularly in the debate over moral nativism, in which the psychological nature of human beings is the topic of debate, information about the way the mind works is of immeasurable worth. With this in mind, the nativist/antinativist debate may be more easily nativist/antinativist debate may be
resolved than previously imagined.

resolved than previously imagined.

Certain subgroups of the population, many first-generation immigrants for example, are in the unusual position of being raised with one set of values and then living their adult lives in a society holding a completely different set of values. This situation allows the existence of a moral critical period to be experimentally tested. Very different predictions will be made about the intuition of immigrants depending on the existence of the moral critical period.

Let us for the moment assume that no critical
Lextention Let us for the moment assume that no critical period exists. After leaving their home country, first-generation immigrants will be exposed to new homes. After living in the host society for ome time having the opportunity to reflect on their experiences immigrants should come to dopt many of the vic adopt many or the vews of their new culture. One would expect the change in their moral
attitudes to be positively correlated with the amount of time spent in their host country.

Now let us imagine that the linguistic analogy holds and morality is innate. Immigrants who left their home country as young children before the linguistic critical period has passed speak the language of their host country without any trace of an accent. As the age at which an individual immigrates increases, however, so does the strength of the individual's accent In much the same way, one would expect the presence or absence of a "moral accent" reminiscent of the immigrant's home country to be highly correlated with the age at which immigrate at younger ages would be expected to be much more acculturated to the new society's values than older immigrants, regardless of the length of time spent in the host country.

An experiment could be designed using the vignette from the beginning of this paper. Indian and American subjects, as previously noted, responded differently to the story ${ }^{12}$ These differences are broadly reflective of difference differis and pessonal saisfacton while Indins greater appreciation of duty and familial loyalty What, then, of Indian-American immigrants? Confirming or refuting the existence of a mora critical period may be as simple as repeating Miller and Bersoff's experiment among the Indian-American immigrant community.

For clarification, it is useful to consider a scenario in which an Indian family leaves its native country and they become American immigrants. If there is a moral critical period then those family members who have passed this stage when they leave their home country will continue to reflect the values of their native culture, while the younger generation will adop typically American values. Speaking in the mode of the linguistic analogy, the older generation will retain a "moral accent" typical of Indians 


\section{Morality with an Accent}

\section{Conclusions}

Innovative researchers in various fields have dependence on empirical data, is a fruitful direction contributed greatly to the field of ethics. The for philosophical inquiry. The study proposed in debate over nativism sparked by Chomsky this paper has the potential to solve a philosophical and later applied to morality is one example of puzzle irresolvable by a priori reasoning alone. By the value to philosophy of drawing from other expanding their arsenal beyond abstract reasoning disciplines. As has been shown in this a expanding their assenal beyond abstract reasoning disciplines. As has been shown in this article, to include experimentation, philosophers are able evidence both for and against moral nativist to approach difficult problems from interesting new
theory is strong, advanced in large part by angles, often finding solutions to age-old debates. 